\title{
Other Problem Visual Analogue Scale
}

National Cancer Institute

\section{Source}

National Cancer Institute. Other Problem Visual Analogue Scale. NCI Thesaurus. Code C141634.

A scale for reporting another problem, which ranges from 0 to 10 , on which the patient marks the current level of the other problem experienced. 